\title{
Long Non-Coding RNA H19 Acts as an Estrogen Receptor Modulator that is Required for Endocrine Therapy Resistance in $\mathrm{ER}^{+}$Breast Cancer Cells
}

\author{
Pratima Basak ${ }^{a, b, c}$ Sumanta Chatterjee ${ }^{a, b, c}$ Vasudeva Bhat ${ }^{a, b, c} \quad$ Alice Su ${ }^{a}$ \\ Hyerang Jin ${ }^{a}$ Victoria Lee-Wing ${ }^{a}$ Qian Liu ${ }^{d}$ Pingzhao Hu ${ }^{d}$ \\ Leigh C Murphy ${ }^{a, d}$ Afshin Raoufa,b,c
}

${ }^{a}$ Research Institute of Oncology and Hematology, Cancer Care Manitoba, Manitoba, bepartment of Immunology, University of Manitoba, Manitoba, 'Regenerative Medicine Program, University of Manitoba, Manitoba, dDepartment of Biochemistry and Medical Genetics, University of Manitoba, Canada

\section{Key Words}

Tamoxifen Resistance $\bullet$ Fulvestrant $(\mathrm{ICl})$ resistance $\bullet$ Endocrine Therapy resistance $\bullet$ H19 $\bullet$ ER $\alpha$

- Notch signaling $\bullet$ C-MET signaling $\bullet \mathrm{ER}^{+}$breast cancers

\begin{abstract}
Background/Aims: Blocking estrogen signaling with endocrine therapies (Tamoxifen or Fulverstrant) is an effective treatment for Estrogen Receptor- $\alpha$ positive (ER+) breast cancer tumours. Unfortunately, development of endocrine therapy resistance (ETR) is a frequent event resulting in disease relapse and decreased overall patient survival. The long noncoding RNA, H19, was previously shown to play a significant role in estrogen-induced proliferation of both normal and malignant $\mathrm{ER}^{+}$breast epithelial cells. We hypothesized that H19 expression is also important for the proliferation and survival of ETR cells. Methods: Here we utilized established ETR cell models; the Tamoxifen (Tam)-resistant LCC2 and the Fulvestrant and Tam cross-resistant LCC9 cells. Gain and loss of H19 function were achieved through lentiviral transduction as well as pharmacological inhibitors of the Notch and c-Met receptor signaling pathways. The effects of altered $H 19$ expression on cell viability and ETR were assessed using three-dimensional (3D) organoid cultures and 2D co-cultures with low passage tumour-associated fibroblasts (TAFs). Results: Here we report that treating ETR cells with Tam or Fulvestrant increases H19 expression and that it's decreased expression overcomes resistance to Tam and Fulvestrant in these cells. Interestingly, H19 expression is regulated by Notch and HGF signaling in the ETR cells and pharmacological inhibitors of Notch and c-MET signaling together significantly reverse resistance to Tam and Fulvestrant in an $H 19$-dependent manner in these cells. Lastly, we demonstrate that $H 19$ regulates $E R \alpha$

\begin{tabular}{ll}
\hline Afshin Raouf, PhD & Department of Immunology, University of Manitoba \\
& 471 Apotex Centre 750 McDermot Avenue, Winnipeg, R3E 0T5, Manitoba (Canada) \\
& E-Mail Afshin.Raouf@umanitoba.ca
\end{tabular}
\end{abstract}

\section{KARGER}


expression at the transcript and protein levels in the ETR cells and that $H 19$ protects ER $\alpha$ against Fulvestrant-mediated downregulation of $E R \alpha$ protein. We also observed that blocking Notch and the c-MET receptor signaling also overcomes Fulvestrant and Tam resistance in 3D organoid cultures by decreasing ER $\alpha$ and H19 expression in the ETR cells. Conclusion: In endocrine therapy resistant breast cancer cells Fulvestrant is ineffective in decreasing ER $\alpha$ levels. Our data suggest that in the ETR cells, H19 expression acts as an ER modulator and that its levels and subsequently ER $\alpha$ levels can be substantially decreased by blocking Notch and c-MET receptor signaling. Consequently, treating ETR cells with these pharmacological inhibitors helps overcome resistance to Fulvestrant and Tamoxifen.

(c) 2018 The Author(s)

Published by S. Karger AG, Basel

\section{Introduction}

Estrogen signaling plays a central role in regulating the proliferation and survival of estrogen-dependent tumours that make up the majority of breast cancer cases [1]. Estrogen signaling is conducted through estrogen receptor alpha and beta, however ER-alpha $(E R \alpha)$ is thought to play a dominant role in breast carcinogenesis [2]. Upon ligand binding ER $\alpha$ is phosphorylated on several sites resulting in activation of estrogen-regulated genes involved in cell proliferation and survival [3]. Therefore, endocrine therapies such as Tamoxifen (Tam) that acts as a competitive ligand inhibitor of $E R \alpha$ and Fulvestrant (ICI-182, 780, ICI) which leads to ubiquitination and degradation of $E R \alpha$ protein [4-6], are effective measures aimed at targeting $E R \alpha$, thus limiting the proliferation and survival of $E R \alpha^{+}$breast cancer cells. These agents are very effective first and second-line endocrine therapies for all stages of $E R \alpha^{+}$breast tumours, however, a small number of these tumours show de novo resistance or after prolonged treatment acquired resistance to both agents can occur in about a third of initially responsive tumours. The development of endocrine therapy resistance (ETR) leads to cancer progression to metastatic disease therefore decreasing overall patient survival which poses a major clinical challenge [7, 8]. Mechanisms of acquired ETR have frequently been studied in the ER+ human breast cancer cell lines, MCF7 and T-47D. By placing MCF7 cells in ovariectomized immune-deficient mice, a new estrogen-independent cell line was obtained [9]. Additional cell lines that show resistance to Tam (LCC2) or ICI (LCC9) were generated after long-term exposure in vivo to these drugs. Studies using the LCC cell system have revealed that these ETR cells show high expression of ER $\alpha$ and are estrogen independent. Several mutations in the $E R \alpha$ gene have been identified that confer ligandindependent activity of $E R \alpha[10,11]$. Interestingly, decreasing $E R \alpha$ expression in the LCC9 cells results in cell death [12] however, mechanisms that can contribute to maintaining $E R \alpha$ expression in these ETR cells remains unknown.

We recently showed that the estrogen-induced proliferation of normal human ER+ breast epithelial cells requires expression of the $H 19$ gene which is an estrogen-regulated breast oncogene [13]. Loss of H19 expression leads to decreased breast cancer cell proliferation and decreased tumour volume in mice $[14,15]$. H19 is a long non-coding RNA that has been shown to regulate estrogen-induced proliferation of $E R \alpha^{+}$breast cancer cells and we recently showed that $H 19$ also regulates the proliferation of $E R \alpha^{+}$normal human breast epithelial cells [13]. H19 RNA consists of the micro RNA675 (miR675) in its first exon which regulates cell proliferation by suppressing retinoblastoma $(R B)$ gene expression [16]. As well, the exons 2-5 fragment of $H 19$ limits the bioavailability of Let-7 microRNA, leading to increased cell proliferation $[17,18]$. Based on the important role that $H 19$ plays in regulating proliferation of normal and malignant $E R \alpha^{+}$breast cells, we hypothesize that $H 19$ plays an equally important role in maintaining the ETR in therapy refractory tumours.

In this report, we demonstrate that decreasing H19 expression either by lentiviral transduction or by using pharmacological inhibitors that block Notch and c-MET receptor signaling, sensitizes the ETR cells to Tam and ICI in an H19-dependent manner. We further show that $H 19$ modulates $E R \alpha$ expression in the ETR cells and might protect these cells against ICI-mediated cell death. The delivery of interfering $R N A$ fragments to treat breast cancer 


\section{Cellular Physiology Cell Physiol Biochem 2018;51:1518-1532 \\ and Biochemistry \begin{tabular}{l|l} 
Dublished online: 29 November 2018 & $\begin{array}{l}\text { (c) } 2018 \text { The Author(s). Published by S. Karger AG, Basel } \\
\text { www.karger.com/cpb }\end{array}$ \\
\hline Basak et al. $H 19$ is Required For Endocrine Therapy Resistance in ER + Breast Cancer Cells
\end{tabular}}

patients has been challenging in the clinic. Therefore, based on our data, pharmacological inhibitors that block Notch and c-MET receptor signaling in combination with endocrine therapies might prove to be effective treatment options for patients with recurrent $E R \alpha^{+}$ breast cancer.

\section{Materials and Methods}

Breast cancer cells

The Tamoxifen (Tam)-resistant LCC2 and the Fulvestrant and Tam (ICI 182, 780, ICI, Tam) crossresistant LCC9 cells were obtained from Dr. Robert Clarke (Georgetown University, Washington, DC) at low passage and were routinely maintained in phenol-red free (PRF) Improved Minimum Essential Medium (IMEM) (Thermo Fisher Scientific, Waltham, USA) supplemented with 5\% charcoal-stripped serum (CSS, v/v) $2 \times$ charcoal/dextran-treated fetal bovine serum, FBS, (estrogen-depleted growth media) and $100 \mathrm{nM}$ 4-hydroxytamoxifen (4-OHTam) or $100 \mathrm{nM} \mathrm{ICI}$ as described before [9]. The genetic relationship of the cell lines with the parental MCF-7 cell line was confirmed previously by DNA fingerprinting. ER $\alpha^{+}$MCF7 cells (originally obtained from Dr. McGuire (San Antonio, Texas [19]) and T-47D cells (originally obtained from Dr. Dean Edwards (Baylor College of Medicine, Houston)[20] have been maintained in the current laboratory for $>20$ years. These cells were authenticated on October, 2016 using STR analyses (Genetica Cell Line Testing, Labcorp, Burlington, NC, USA). All experiments were carried out on cells between passages 5-20. MCF7 were maintained in Dulbecco's Modified Eagle Medium (DMEM) supplemented with 5\% FBS and T-47D cells were maintained in Roswell Park Memorial Institute (RPMI)-1640 media (Sigma) supplemented with bovine Insulin (Sigma) and 10\% FBS.

For some experiments, breast cancer cells were cultured to $60 \%$ confluency and treated with ICI (at the indicated doses), or 4-OHTam (100 nM) or a pan Notch signaling inhibitor R04929097 (RO, at the indicated doses) or the c-MET receptor selective inhibitor Tivantinib (ARQ 197, TIV, at the indicated doses). Both inhibitors were from Selleckchem (Houston, TX, USA). To treat cells with a combination of these signaling inhibitors, LCC2, LCC9 or T-47D $\mathrm{D}^{\text {ICl-Res }}$ or T-47 $\mathrm{D}^{\text {Tam-Res }}$ were grown to $60 \%$ confluency and treated with a combination of RO and TIV or RO or TIV and ICI or Tam or vehicle control for 1 or 3 days.

\section{Gain and loss of H19 function in breast cancer cells}

The full length H19 cDNA was purchased from Dharmacon (Colorado, USA) and was sequence verified. $H 19$ cDNA was sub-cloned into pHIV-dTomato lentiviral vector (Addgene, Cambridge, MA, USA) [pHIV-dTomato was a gift from Bryan Welm to Addgene (Addgene plasmid \# 21374)] and used to produce lentivirus which was then used to infect sub-confluent cultures of LCC9 (LCC9 dTOM H19FL) and LCC2 (LCC2 dTOM H19FL). Some cells were infected with empty dTomato (dTOM) expressing virus as controls. The transduced $\mathrm{dTOM}^{+}$breast cancer cells were sorted by a flow cytometer and further expanded for future experiments. To knockdown $H 19$ expression in the resistant breast cancer cells, a pool of 3 different pGIPZpuro-GFP plasmids containing short hairpin (sh) RNA targeting the H19 RNA or a pGIPZ-puro-GFP vector (Thermo Fisher Scientific, Waltham, USA) expressing a scrambled sh-RNA fragment were used to generate lentivirus and then used to infect LCC2 and LCC9 cells. All cells were infected using a previously described protocol [13, 21]. Briefly, $5.5 \times 10^{5}$ cells were plated in $60 \mathrm{~mm}$ culture plates in PRF-IMEM supplemented with $5 \%$ CSS-FBS for $24 \mathrm{hrs}$ and then infected with $5-7 \times 10^{6}$ lentiviral particles for $6 \mathrm{hrs}$. After 4 days, the transduced $\mathrm{GFP}^{+}$breast cancer cells were sorted by a flow cytometer and expanded for future experiments. The successful knockdown or overexpression of $H 19$ gene in the transduced cells were assessed using qPCR. NOTCH4-ICD (a constitutively active form of NOTCH4) cDNA carrying plasmid (MIY-NOTCH4-ICD) construct was a kind gift from Dr. Aly Karsan (Genomic Sciences Centre, B.C. Cancer Agency, Vancouver, Canada). The cDNA was cloned into lenti-viral vector KA391, sequence verified and used to produce lentivirus to infect the LCC9 cells as described. Empty KA391 vector was used as control. The transduced (GFP+) LCC9 cells were isolated using flow cytometry. All cells were transduced with separate lentivirus to produce 3 independent biological replicates. 


\section{Cellular Physiology Cell Physiol Biochem 2018;51:1518-1532 and Biochemistry \begin{tabular}{l|l} 
DOI: $10.1159 / 000495643$ & \\
(c) 2018 The Author(s). Published by S. Karger AG, Basel
\end{tabular}

\section{Intracellular Flow cytometry}

The transduced shH19-LCC2 and shH19-LCC9 or scramble-LCC2/-LCC9 cells were grown in 5\% CSSPRF medium for $48 \mathrm{hrs}$ and treated with RO (250 $\mu \mathrm{M})$, TIV (50 nM), ICI (100 nM), 4-OHTam (100 nM), or combinations of these inhibitors for an additional $24 \mathrm{hrs}$. Subsequently, single cell suspensions were fixed and permeabilized using BD Perm and Fix Kit (BD Biosciences, San Jose, CA, USA) as described before [13]. Fixed cells were then stained with anti $E R \alpha$ antibody (6F11, Abcam), and $E R \alpha$ expression was detected using an anti-mouse phycoerythrin (PE, Biolegend, San Diego, CA, USA) secondary antibody via flow cytometry. FlowJo software (Ashland, Oregon, USA) was used to obtain the Median Fluorescence Intensity (MFI) for all treatment groups and also for those treatment groups that specifically contained GFP+ cells.

\section{Breast cancer cell-fibroblast co-cultures}

The LCC2 and LCC9 cells $\left(5 \times 10^{4}\right)$ were either cultured alone or placed in co-culture with tumourassociated fibroblasts (TAFs, $5 \times 10^{4}$ ) using 12-well culture plates. TAFs were obtained from primary $E R \alpha^{+}$ human breast tumours as described before [22]. Primary human breast tumours were obtained from informed consented patients in accordance with the University of Manitoba Research Ethics Board (REB\# HS14919). TAFs were assessed to lack EpCAM (StemCell Technologies, Canada), CD45 (Biolegend, San Diego, USA), and CD31 (eBioscience, ThermoFisher Scientific, USA) expression but had strong $\alpha$-smooth muscle actin and fibroblast specific protein 1 expression. Therefore, EpCAM expression was used to determine breast cancer cell numbers in the co-cultures. After $48 \mathrm{hrs}$, cells were treated with RO $(250 \mu \mathrm{M})$, TIV (50 nM), and ICI (100 nM) or 4-OHTam (100 nM) or vehicle control for $24 \mathrm{hrs}$. Subsequently singlecell suspensions were stained with an anti EpCAM antibody and the number of viable (propidium iodide negative, $\mathrm{PI}^{-}$) EpCAM+ cells was determined by flow cytometry.

\section{Cell viability assay}

Cancer cell viability was measured using the colorimetric water-soluble tetrazolium salt cell counting kit-8 (WST-8) assay (Dojindo Molecular Technologies, Rockville, MD, USA) as per manufacturer's protocol. Briefly, cells $\left(1 \times 10^{4}\right)$ were seeded in each well of a 96-well plate for $24 \mathrm{hrs}$ and then growth medium was supplemented with different concentrations of ICI, 4-OHTam, RO, TIV or their combinations or vehicle control. Subsequently, cells were treated with the WST-8 reagent and after solubilisation of the purple formazan crystals, absorbance was measured at $450 \mathrm{~nm}$ (background wavelength, $650 \mathrm{~nm}$ ) using a plate reader (Synergy H1 Hybrid Reader, BioTek, Vermont, USA).

\section{Organoid Cultures}

LCC2 and LCC9 cells $\left(5 \times 10^{4}\right)$ were plated into each well of 96-well plates containing $50 \mu \mathrm{L}$ of polymerized, growth factor-reduced and PRF matrigel (BD Biosciences, San Jose, CA, USA). After 48 hr, the organoid-like structures were either treated with ethanol (vehicle control) or RO (250 $\mu \mathrm{M})$, TIV (50 nM), and ICI (100 nM) or Tam (100 nM). After 7 days, cell viability was determined by WST-8 assay as described.

\section{Statistical analysis}

For all experiments, data were generated from at least three independent replicates and were expressed as average \pm standard deviation (SD). Two-tailed Student's t-tests were used to determine statistical significance and for multi-pairwise comparisons ANOVA was used. All tests were done using GraphPad Prism 7 program (La Jolla, CA, USA).

\section{Results}

H19 regulates resistance to ICI and Tam in ETR cells

To assess if $H 19$ expression is important to the development of endocrine therapy resistance, therapy sensitive MCF7 cells were cultured in estrogen-deprived growth conditions and exposed to ICI for up to 12 days and $H 19$ expression was quantified (Fig. 1A). Exposure to ICI initially resulted in decreased $H 19$ expression after 24 hrs. Subsequently, H19 expression was significantly increased in the MCF7 cells after 3 days of ICI exposure and its high expression was sustained for up to 12 days, suggesting that $H 19$ expression 


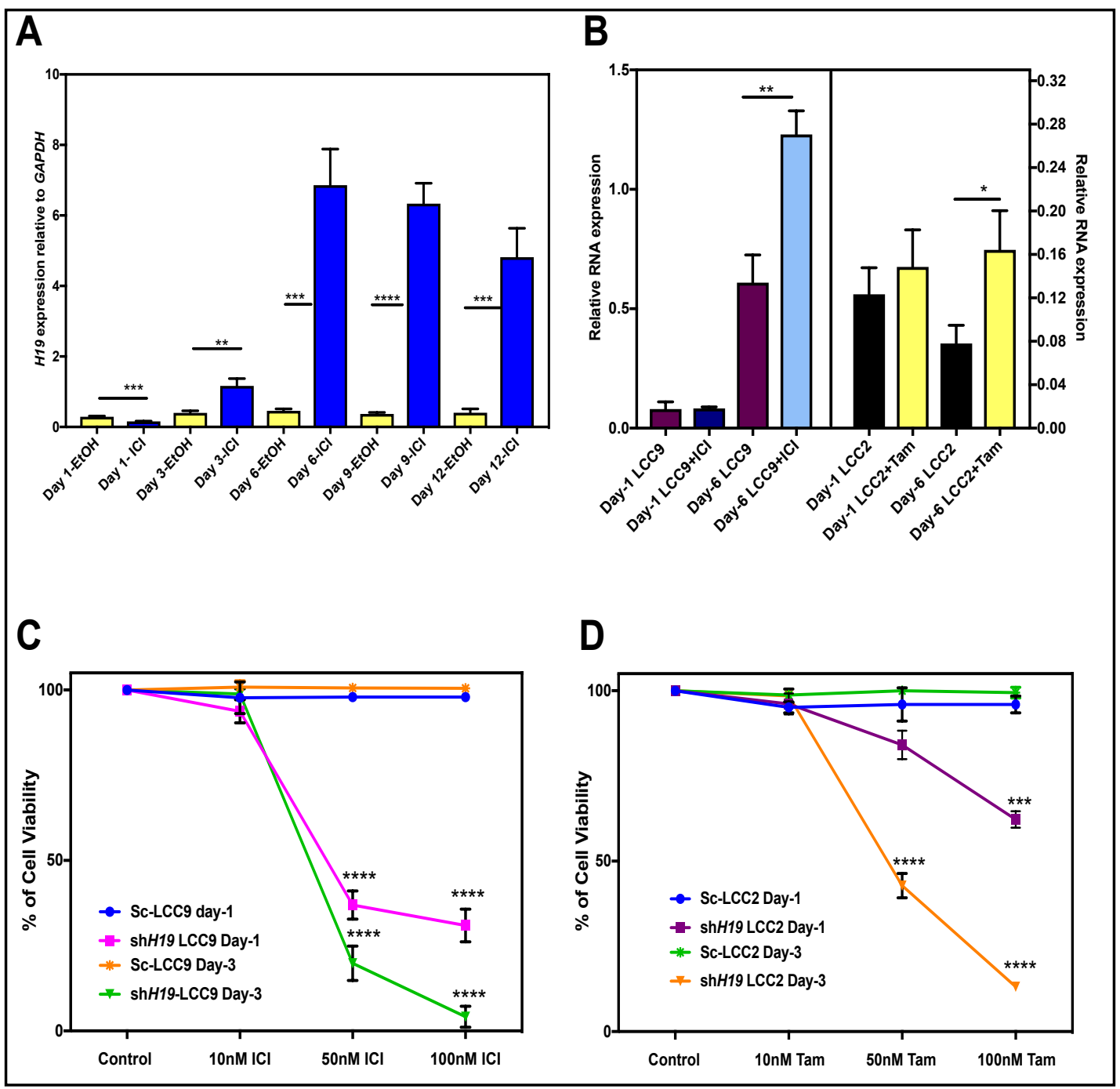

Fig. 1. H19 expression is increased in breast cancer cells treated with endocrine therapies. (A) MCF7 cells were cultured in estrogen-depleted growth media and treated with ICI or vehicle control (ethanol) up to 12 days. H19 expression in the treated cells was determined by qPCR on the indicated days. (B) LCC9 and LCC2 were treated with Fulvestrant (ICI) or 4-hydroxytamoxifen (Tam). H19 expression was determined using qPCR after 1 and 6 days. In both cases, H19 expression was normalized to the GAPDH transcript levels and average transcript expression and standard deviation (SD) from 3 independent experiments are shown as bar graphs. H19 RNA levels were decreased in the LCC9 (C) and LCC2 (D) cells with shRNA using the lentiviral transduction (shH19LCC9, shH19LCC2). Scrambled shRNA expressing cells were used as controls (Sc-LCC9 and Sc-LCC2). Impact of ICI and Tam on viability of the transduced cells was measured relative to ethanol-treated cells (set to 100\%). Average cell viability from at least 3 independent experiments and SD are shown in the graphs. ${ }^{*} \mathrm{P}<0.05,{ }^{* *} \mathrm{P}<0.005,{ }^{* * *} \mathrm{P}<0.0005,{ }^{* * * *} \mathrm{P}<0.0005$.

might be associated with the development of endocrine therapy resistance. To test this hypothesis, $H 19$ expression was examined in the Tam and ICI cross-resistant LCC9 and the Tam-resistant LCC2 cells. Interestingly, we observed a significant increase $(2.05 \pm 0.31$ fold $)$ in H19 expression in LCC 9 cells treated with ICI after 6 days compared to the untreated control cells (Fig. 1B). In the case of LCC2 cells, treatment with Tam was required to maintain high expression of $H 19$ (2.24 \pm 0.8 fold) (Fig. 1B). Next, we examined if increased $H 19$ expression was required for the Tam- and ICI-resistant phenotype in these cells. For this purpose, we measured cell viability in LCC9 and LCC2 cells transduced with lentivirus expressing a short 


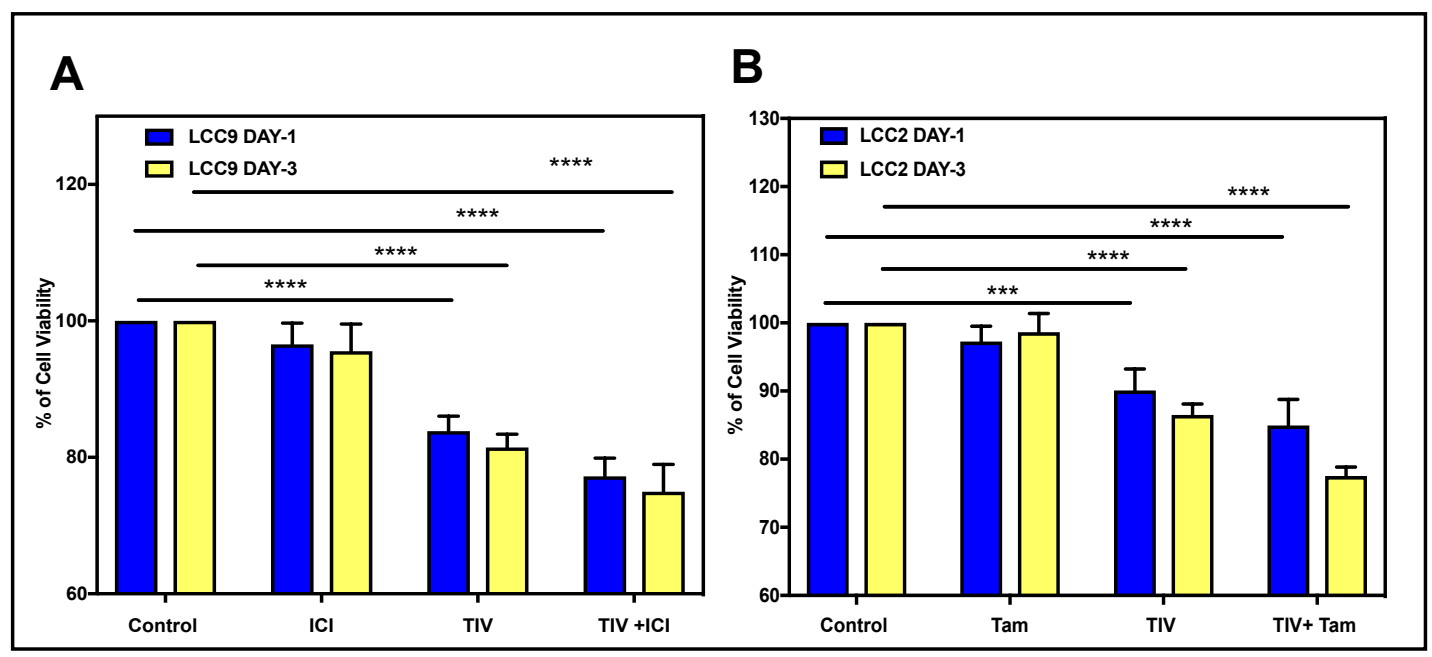

Fig. 2. c-MET signaling is not a major contributor to cell viability of ETR cells. LCC9 (A) and LCC2 (B) cells were treated with Tivantinib (TIV) and cell viability was measured after 1 and 3 days. Viability of ethanoltreated cells was set to $100 \%$ and average cell viability and SD from at least 4 independent experiments are plotted in the bar graphs. ${ }^{* * *} \mathrm{P}<0.0001,{ }^{* * * *} \mathrm{P}<0.0005$.

hairpin RNA (shRNA) against H19 RNA (shH19, Fig. S1A - for all supplemental material see www.karger.com/10.1159/000495643). Decreased H19 expression alone had minimal effects on LCC9 and LCC2 cell viability (Fig. 1C-D). However, when shH19-LCC9 or -LCC2 cells were treated with ICI or Tam for just one day, we observed a significant decrease in cell viability. After 3 days of treatment with ICI, only $4.15 \pm 3 \%$ of shH19-LCC9 cells was viable. Compared to the shH19-LCC9, the LCC2 cells showed less sensitivity to Tam with decreased $H 19$ levels. However, after 3 days of Tam treatment, shH19-LCC2 cell viability was reduced to $13.12 \pm 0.6 \%$ (Fig. 1D). These data indicate that $H 19$ plays a role in maintaining the ETR phenotype and decreasing its expression overcomes resistance to ICI and Tam. To determine if $H 19$ expression level is correlated with maintaining the ETR phenotype, the shH19-LCC9 cells with higher H19 expression (LCC9 ${ }^{\text {H19high }}$ ) compared to the cells with lower $H 19$ expression (LCC ${ }^{H 19 l o w}$ ) were separated based on their GFP expression (Fig. S1B). Interestingly, the ICI effects on decreasing cell viability were only observed when H19 levels were decreased by more than $70 \%$ i.e. in $\left(>70 \%\right.$, LCC $\left.{ }^{H 19 l o w}\right)$ compared to ineffective $H 19$ knockdown (30\%, LCC ${ }^{\text {H19high }}$ ) (Fig. S1C).

\section{MET receptor signaling regulate $H 19$ expression in ETR breast cancer cells}

Since decreasing $H 19$ expression in LCC 9 and LCC2 cells helped overcome their resistance to ICI and Tam, we considered signaling pathways that might regulate $H 19$ expression in these ETR cells and whether blocking such signaling pathways would overcome their resistance to endocrine therapies. Previous reports have indicated that HGF-c-MET signaling regulates H19 expression [23, 24]. Having established that the LCC9 and LCC2 cells express c-MET receptor (Fig. S2A), we determined if c-MET receptor signaling regulated H19 expression in these ETR cells. Treatment with 50nM of a c-MET inhibitor, Tivantinib (TIV) was sufficient to reduce $H 19$ expression by $2.61 \pm 0.74$ fold (Fig. S2B), with only a marginal effect on LCC9 and LCC2 cell viability after 3 days $(81.43 \pm 1.95 \%$ and $86.47 \pm 1.22 \%$ respectively, Fig. 2A-B). As well, in combination with ICI or Tam, TIV treatment only marginally affected LCC 9 and LCC2 cell viability (down to $74.97 \pm 4 \%$ and $77.51 \pm 3.49 \%$ accordingly) (Fig. $2 \mathrm{~A}-\mathrm{B}$ ). This finding was consistent with our observation that greater than $70 \%$ decrease in $H 19$ expression is required to overcome resistance to ICI. These data suggest that other signaling pathways are also involved in regulating $H 19$ expression in these ETR breast cancer cells. 

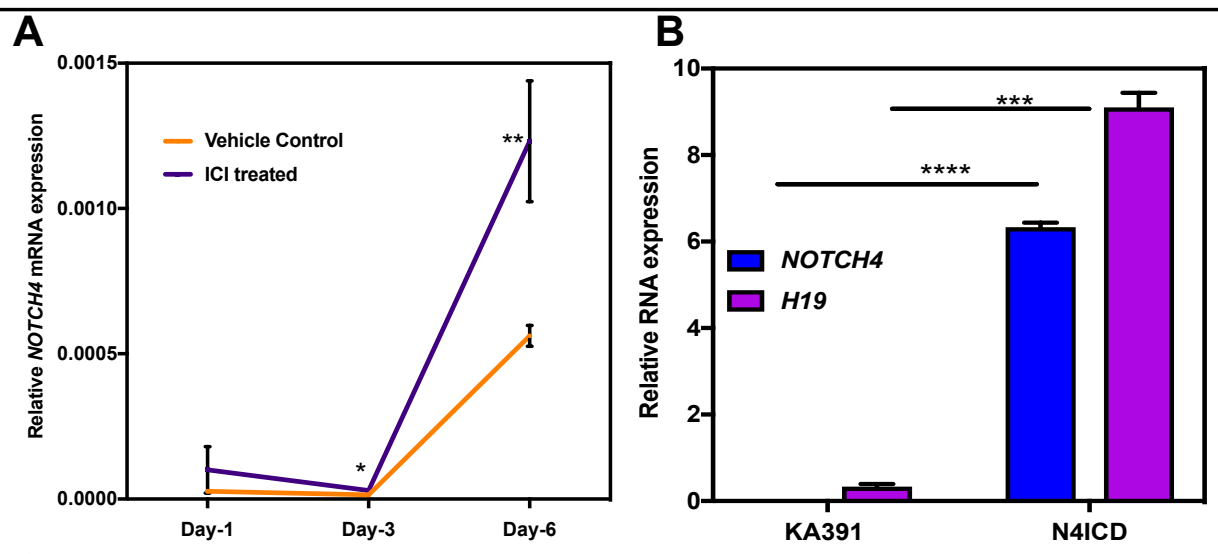

C
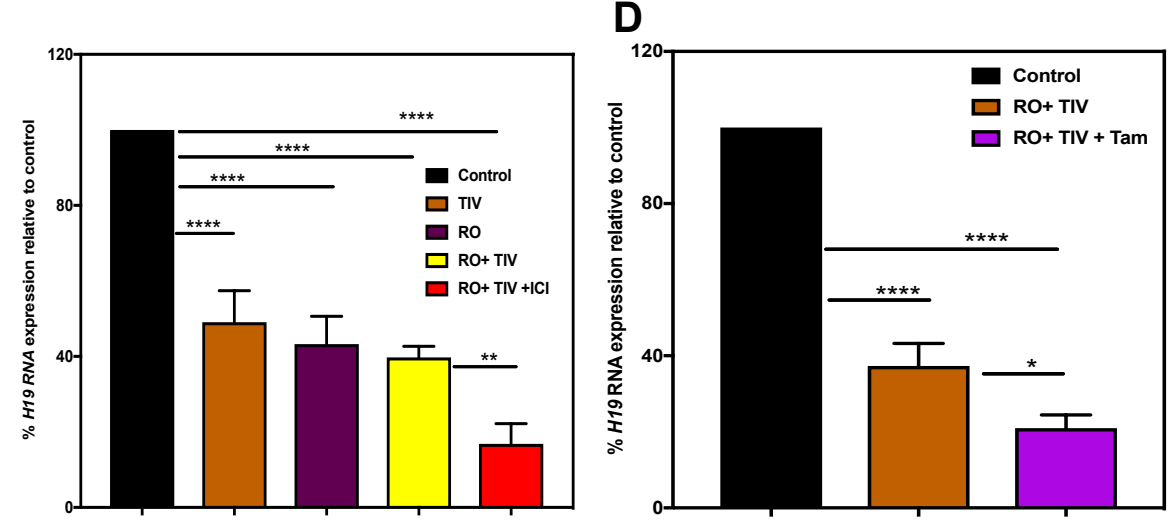

$\mathbf{E}$

$\mathbf{F}$
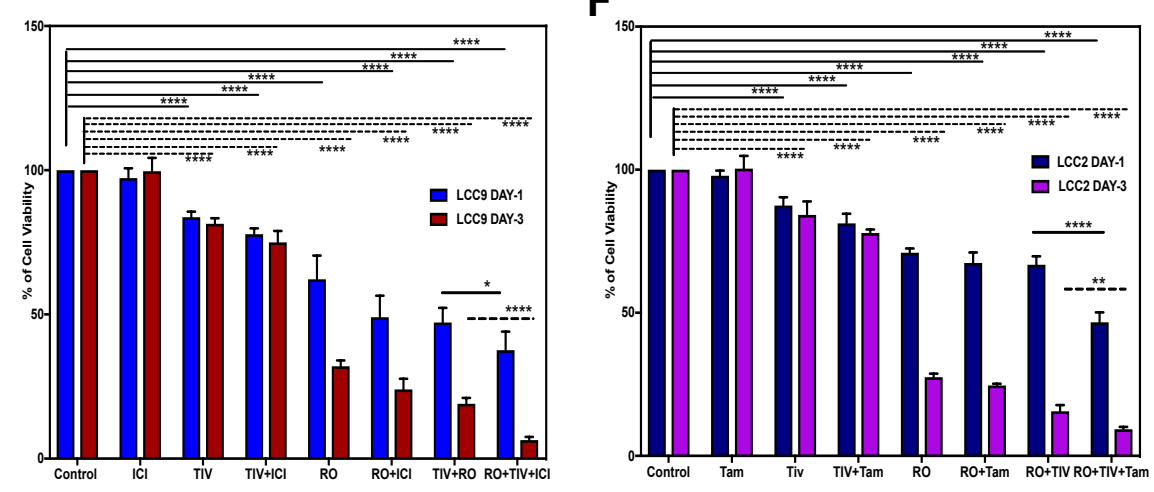

Fig. 3. Notch signaling regulates H19 expression in the endocrine therapy resistant cells. (A) LCC9 cells were treated with Fulvestrant (ICI) or ethanol as vehicle control and RNA was collected on different days. NOTCH 4 transcript levels were determined by qPCR and normalized to the GAPDH transcript levels. Average transcript levels and SD from 3 independent experiments are shown. (B) LCC9 cells were transduced by lentivirus to express a constitutively active form of NOTCH 4 or GFP as control. NOTCH4 and H19 transcript levels were determined by qPCR and normalized to the GAPDH transcript levels. Average expression and SD from 3 independent experiments are plotted. LCC9 (C) or the LCC2 (D) cells were treated with Tivantinib (TIV, $50 \mathrm{nM})$ or RO $(250 \mu \mathrm{M})$, or TIV+ RO in combination +/- Fulvestrant (ICI, 100nM) or Tam $(100 \mathrm{nM})$ for $24 \mathrm{hrs}$. H19 expression was determined by qPCR and normalized to the GAPDH transcript levels and H19 expression in the controls is set to 1 . Average expression and SD from 3 independent experiments are shown. LCC9 (E) and LCC2 (F) cells were treated with RO and TIV as single agents, or in combination +/- ICI or Tam for 1 and 3 days and cell viability was measured. Viability of the vehicle control-treated cells was set to $100 \%$. Average cell viability and SD from at least 4 experiments are plotted in the bar graphs. ${ }^{*} \mathrm{P}<0.05$, ${ }^{* *} \mathrm{P}<0.005,{ }^{* * *} \mathrm{P}<0.0001,{ }^{* * * *} \mathrm{P}<0.0005$. 
NOTCH4 and c-MET signaling together contribute to H19-dependent resistance to endocrine therapies

Previous reports suggested that Notch signaling, in particular Notch Receptor 4, NOTCH4, contributes to the ETR phenotype through enhanced epithelial to mesenchymal transition as well as regulating cancer stem cell activity $[25,26]$. Interestingly, we observed that NOTCH4 transcript levels were significantly increased in ICI-treated LCC 9 cells after 6 days (Fig. 3A). Furthermore, forced expression of a constitutively active form of NOTCH4, significantly increased $H 19$ expression in these cells (Fig. 3B). Next, we determined that $250 \mu \mathrm{M}$ of R04929097 (RO), a pan Notch signaling inhibitor was sufficient to significantly decrease H19 and HES1 (a known NOTCH target) transcript levels $(2.66 \pm 0.56$ and $2.71 \pm 1.44$

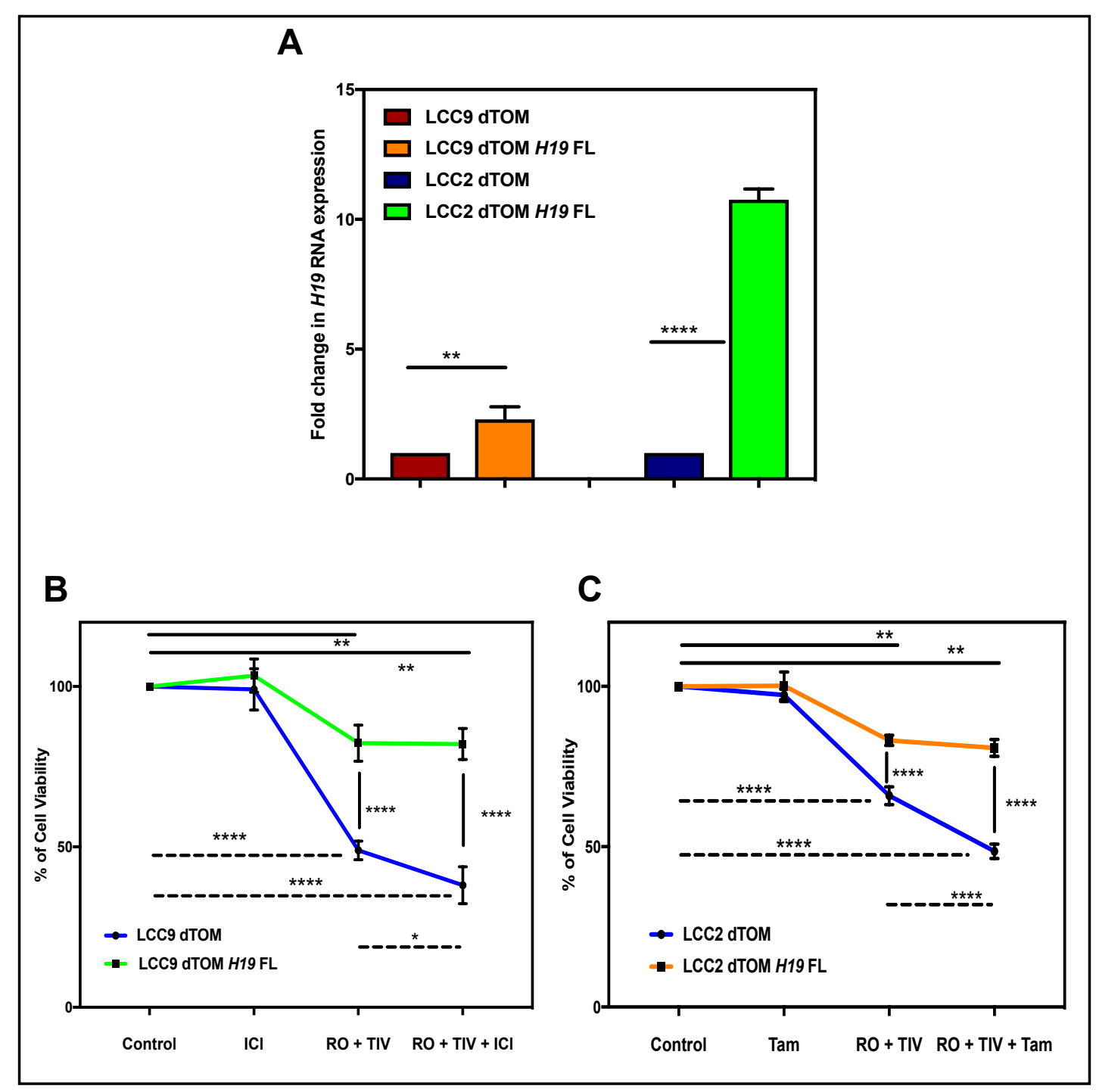

Fig. 4. Constitutive overexpression of H19 protects ETR cells against sensitization to endocrine therapy in the presence of Notch and c-MET signaling blockers. (A) Lentiviral transduction was used to force expression of full-length H19 (dTOM H19FL) or empty vector dTOM in LCC9 and the LCC2 cells. H19 expression was determined and normalized to the GAPDH transcript levels and the values are represented as fold changes. Average transcript levels and SD from 3 independent experiments are shown. (B) LCC9-dTOM H19FL and (C) LCC2 dTOM H19FL cells were treated with ICI alone, or Tam alone, or RO + TIV with or without ICI or Tam for 24 hrs and cell viability was measured. Viability of vehicle control-treated cells was set to $100 \%$. Average viability and SD from at least 3 separate experiments are plotted in the line graphs. ${ }^{*} \mathrm{P}<0.05$, ${ }^{* *} \mathrm{P}<0.005, * * * * \mathrm{P}<0.0005$. 
fold respectively) in the LCC9 cells (Fig. S3A). Interestingly however, RO treatment of the ICI/Tam-sensitive MCF7 cells had no effect on H19 expression while HES1 transcript levels were decreased (Fig. S3B). The most significant decrease in $H 19$ expression was observed when LCC9 and LCC2 cells were treated with a combination of RO, TIV and ICI or Tam (Fig. 3C-D) where $H 19$ expression was decreased by $83.14 \pm 5.33 \%$ and $79.03 \pm 3.49 \%$ respectively. RO as a single agent was more effective than TIV or TIV+ICI in decreasing LCC9 and LCC2 cell viability after 3 days $(32.02 \pm 01.99 \%$ and $27.47 \pm 1.28 \%$ respectively, Fig. $3 \mathrm{E}-\mathrm{F})$ however, RO was unable to overcome resistance in ETR cells treated with ICI or Tam for 3 days (nonsignificant decrease to $23.98 \pm 3.73 \%$ and $24.57 \pm 0.63 \%$ respectively). In combination with TIV, RO decreased LCC9 and LCC2 cell viability further $(19.03 \pm 2.02 \%$ and $15.59 \pm 2.16 \%$ respectively) after 3 days. However, the most significant decrease in LCC9 and LCC2 cell viability was observed in cells treated with the combination of RO, TIV, and ICI or Tam (decreased to $6.40 \pm 1.15 \%$ and $9.29 \pm 0.86 \%$ respectively), suggesting that blocking Notch and c-MET receptor signaling together helps overcome ICI and Tam resistance in the ETR breast cancer cells. We also found similar results in the ICI- and Tam-resistant T-47D (T47D ICI-Res, T47D Tam-Res) cells, indicating that our observations are not limited to the LCC9 and LCC2 cells (Fig. S3C). Of note, even though TIV and RO are equally effective in decreasing $H 19$ expression $(<70 \%)$ as single agents in the LCC9 cells (Fig. 3C), RO has a larger effect on decreasing cell viability compared to TIV (Fig. 3E-F).

Our data so far indicate that sensitization of LCC9 and LCC2 cells to ICI and Tam when treated with Notch and c-MET receptor inhibitors might require decreased H19 expression. To test this hypothesis, LCC9 and LCC2 cells were transduced with lenti-virus to express full-length $H 19$ (Fig. 4A) and the transduced cells were treated with a combination of RO, TIV and ICI or Tam (Fig. 4B-C). Interestingly, the $H 19$ overexpressing ETR cells remained resistant to ICI and Tam when treated with combination of RO and TIV. However, RO and TIV treatment decreased cell viability in $H 19$ overexpressing LCC 9 \& LCC 2 cells by $17.65 \pm 5.61 \%$ and $16.83 \pm 1.56 \%$ (Fig. 4B-C), suggesting that Notch and MET signaling contributes to ETR cell viability via H19-dependent and -independent mechanisms. However, the H19dependent cell survival mechanisms play a more significant role than the H19-independent mechanisms. These observations together indicate that blocking Notch and c-Met receptor signaling together overcome resistance to ICI and Tam in ETR breast cancer cells in an H19dependent manner.

\section{$H 19$ regulates ERa expression in ETR breast cancer cells}

So far, our data indicate that significantly reduced $H 19$ expression $(>70 \%)$ sensitizes LCC9 and LCC2 cells to ICI and Tam, suggesting that ligand-independent ER $\alpha$ signaling is important to the survival of these ETR cells. We therefore examined $E R \alpha$ expression in LCC9 cells showing effective $H 19$ knockdown (>70\%, LCC ${ }^{H 1910 w}$ ) or ineffective $H 19$ knockdown (30\%, LCC $\left.{ }^{H 19 h i g h}\right)$ and found that ER $\alpha$ protein and transcript levels were both significantly decreased in the LCC ${ }^{H 19 l o w}$ cells but not in the LCC ${ }^{H 19 \text { high }}$ compared to control cells (Fig. 5A-B and Fig. S4B). Addition of ICI for 24 hours further decreased $E R \alpha$ protein expression in the LCC9 ${ }^{H 19 l o w}$ cells (Fig. 5A), suggesting that $H 19$ expression protects $E R \alpha$ from ICI-mediated protein degradation. Similar results were found using the LCC2 ${ }^{\text {H19low }}$ cells (Fig. S4A). Although statistically significant, the decreased ER $\alpha$ protein levels in the LCC $2^{\text {H19low }}$ cells was small and not effected further by Tam as is consistent with Tam's mechanisms of action as a competitive inhibitor and not a down-regulator of ER $\alpha$. However importantly, treating LCC9 and LCC2 cells with a combination of RO, TIV, and ICI or Tam significantly decreased ER $\alpha$ expression in these ETR cells (Fig. 5C-D). Interestingly, we found that knocking down ER $\alpha$ in the LCC9 cells also decreased $H 19$ expression (Fig. S4C) providing further support to the notion that ER $\alpha$ signaling is regulated primarily in a ligand-independent manner in the LCC9 cells. 
Fig. 5. H19 regulates $\mathrm{ER} \alpha$ expression in the endocrine therapyresistant breast cancer cells. H19 expression was decreased $>70 \%$ in the LCC9 (LCC9 ${ }^{\text {H19low) }}$ (A) or $<70 \%$ LCC $^{\text {H19high }}$ (B) cells and $\mathrm{ER} \alpha$ protein expression was determined by immunofluorescence and flow cytometry. $\mathrm{R}$ e p r e s e $\mathrm{n}$ t a $\mathrm{t}$ i ve histograms are shown and median fluorescence intensities with SD from at least 5 independent experiments are shown in bar graphs. $\mathrm{ER} \alpha$ expression was measured in LCC9 (C) and LCC2 (D) treated with RO, TIV, and ICI or Tam for 24 hrs using immunofluorescence and flow cytometry. R e p res en t a t i ve histograms are shown and mean fluorescence intensities and SD from 3 independent experiments are shown in bar graphs. $* \mathrm{P}<0.05, \quad * * \mathrm{P}<0.005$, $* * * * \mathrm{P}<0.0005$.
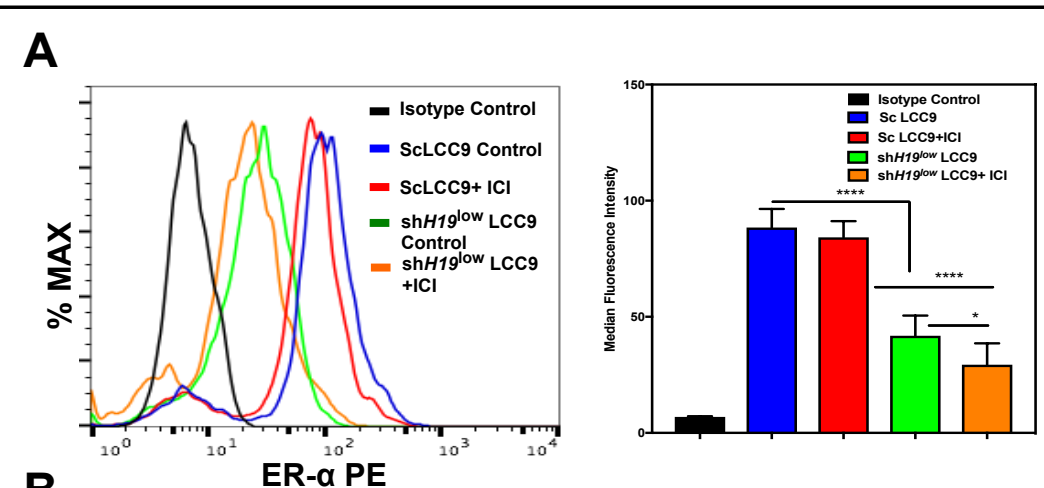

B
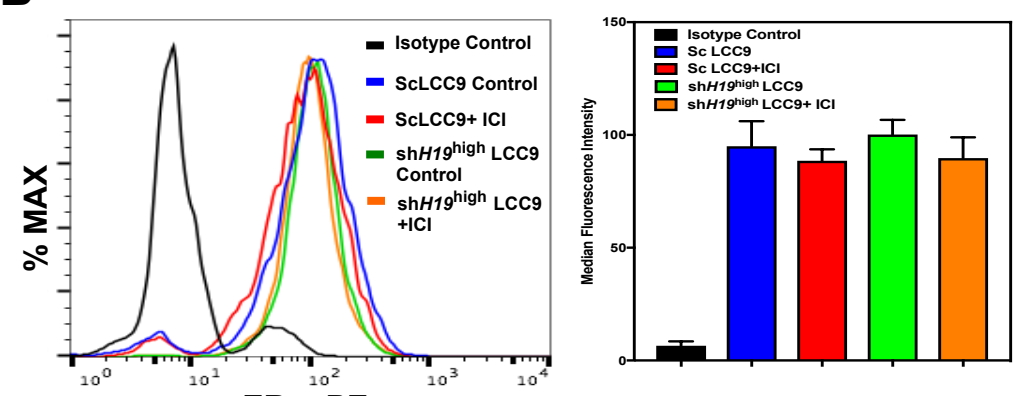

C
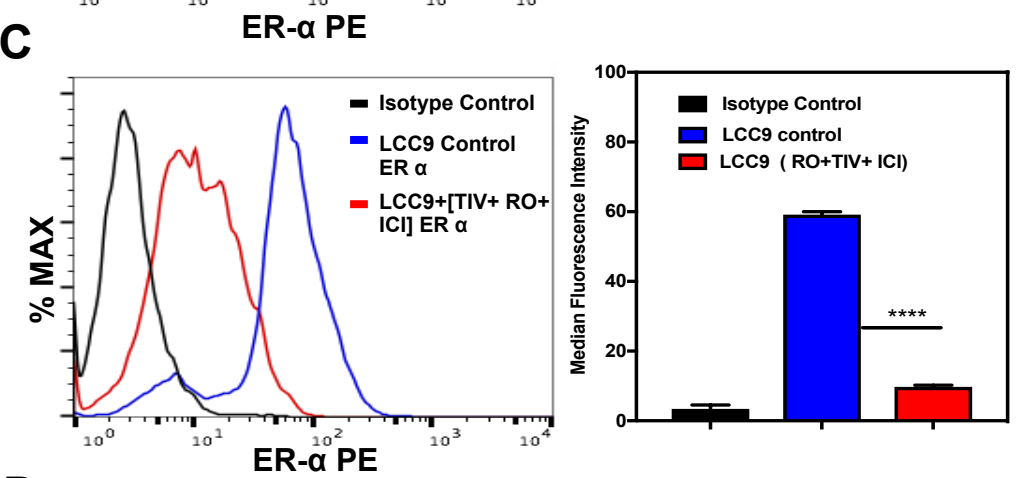

D

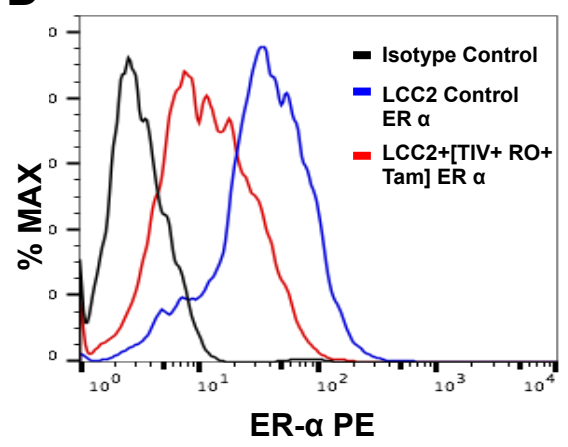

Blocking Notch and c-MET signaling sensitizes ETR cells to endocrine therapy in $3 D$ organoid cultures

To examine if the combination of RO and TIV could overcome ICI and Tam resistance in a more in vivo-like environment, LCC9 and LCC2 cells were placed in 3 dimensional (3D) organoid cultures. Live $\mathrm{EpCAM}^{+}$cells were quantified to determine breast cancer cell number in these co-cultures. We observed that in organoid cultures treated with a combination of RO, TIV and ICI or Tam, cell viability was significantly decreased (to $31.06 \pm 5.87 \%$ and $29.79 \pm 7.96 \%$ of untreated controls, respectively, Fig. 6A-C). Recent evidence supports a 


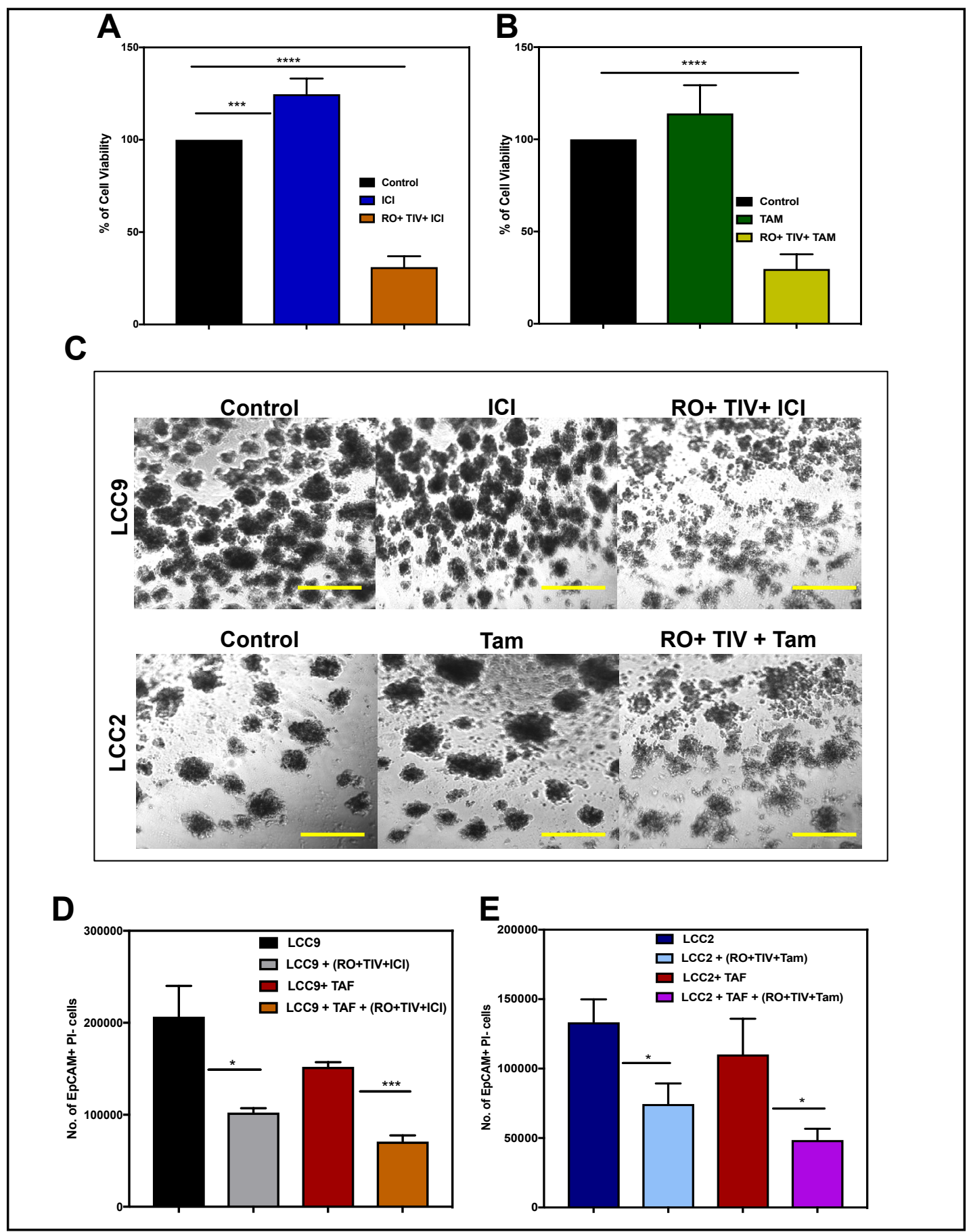

Fig. 6. Blocking Notch and c-MET signaling sensitizes ETR cells to endocrine therapies in 3D organoid cultures. (A) LCC9 and the LCC2 (B) cells were placed in 3 dimensional (3D) matrigel cultures and treated with ICI or Tam alone, or (RO+TIV) with or without ICI or Tam for 24 hrs and cell viability was measured. Viability of vehicle control-treated cells was set to $100 \%$ and average cell viability and SD from 3 independent experiments are shown in the bar graphs. (C) Representative pictures of cells in 3D cultures as in A-B. Scale bar represents $1000 \mu \mathrm{m}$. LCC9 (D) and the LCC2 (E) cells were placed in 2D cultures either alone or in coculture with tumour-associated fibroblasts (TAF) and treated with (RO+TIV) with or without ICI or Tam for $24 \mathrm{hrs}$. The number of Epithelial Cell Adhesion Molecule positive (EpCAM+) propidium iodide negative (PI) cells was determined by flow cytometry and average cell numbers and SD from 3 independent experiments are plotted in the bar graphs. ${ }^{*} \mathrm{P}<0.05,{ }^{* * *} \mathrm{P}<0.0005,{ }^{* * * *} \mathrm{P}<0.0005$.

\section{KARGER}




\section{Cellular Physiology Cell Physiol Biochem 2018;51:1518-1532

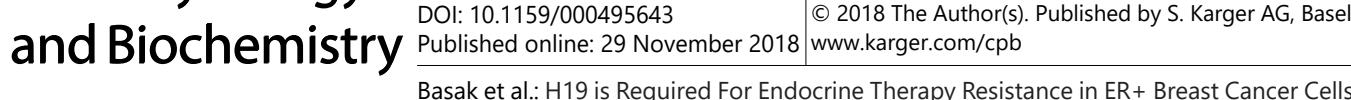

strong role for tumour microenvironment, in particular the tumour-associated fibroblasts (TAFs), as a major modulator of therapy resistance in breast tumours [27, 28]. We therefore examined if blocking Notch and c-MET signaling could sensitize ETR cells to endocrine therapies in the presence of TAFs. For this purpose, LCC9 and LCC2 cells were placed in 2D co-culture with and without TAFs and treated with RO, TIV, ICI or Tam for $24 \mathrm{hr}$. Interestingly, breast cancer cell numbers were significantly decreased in co-cultures treated with RO, TIV, ICI or Tam even more so compared to cancer cell alone cultures (Fig. 6D-E). Lastly, we examined the clinical relevance of $c-M E T, N O T C H 4$, and $H 19$ expression to overall survival of patients with ER+ breast tumours using the TCGA and METABRIC data sets. The transcript expression level for each gene and the combined risk score were binarized into high risk and low risk groups as described in the Supplementary Information. The correlation and differences in overall survival between these risk groups were assessed by Kaplan-Meier analysis. The combined $(H 19+N O T C H 4+M E T)$ high risk score was significantly associated with poorer overall survival in both cohorts (TCGA data set: Hazard Ratio (HR) $=1.90$ (1.113.24), $\mathrm{P}=0.019$; METABRIC data set: $\mathrm{HR}=3.51$ (1.47-8.34), $\mathrm{P}=0.0045)$. These data indicate that high expression of H19, NOTCH4 and MET is associated with poor prognosis in ER+ breast cancers (Fig. S5A-B).

\section{Discussion}

Although ER+ breast tumours respond well to endocrine therapies such as ICI and Tam, over $30 \%$ of these tumours develop resistance or exhibit de novo resistance to such therapies [8]. These recurrent tumours retain ER $\alpha$ expression and yet are refractory to the effects of ICI and Tam. In this study, we report that H19 expression is essential to endocrine therapy resistance in breast cancer cells. We found that Notch and c-MET signaling together regulate H19 expression in the ETR cell line models used in this study. Moreover, pharmacological inhibitors of Notch and c-MET receptor signaling in combination yield significant $(>70 \%)$ decreased $H 19$ expression and overcome resistance to ICI and Tam in these cells in an H19dependent manner. As a non-protein coding gene, $H 19$ poses a significant challenge as a therapeutic target. Therefore, our finding that pharmacological inhibitors of Notch and c-MET receptor signaling overcome resistance in ETR cells through decreased H19-ER $\alpha$ expression could have significant clinical implications. Moreover, we found that $H 19$ regulates $\mathrm{ER} \alpha$ expression and protects ER $\alpha$ from ICI and Tam antagonist action. It is interesting that while ER $\alpha$ levels were reduced in the ETR cells with decreased H19 expression, no effect on cell viability was detected. However, in the presence of ICI, further reduction in ER $\alpha$ expression occurred and this was associated with a significant decrease in cell viability in the LCC9 suggesting that ER $\alpha$ protein in the shH19-ETR cells had become susceptible to ICI-induced protein degradation. A previous report by Cook et al. [12] also indicated that decreased ER $\alpha$ renders LCC 9 cells susceptible to ICI where they observed a 50\% decrease in relative cell density after 3 days of ICI treatment. Based on our finding that $\mathrm{H} 19$ regulation of ER $\alpha$ is reciprocated by ER $\alpha$, we posit that the sensitization of ER $\alpha$-knocked down LCC 9 cells to ICI reported by Cook et. al., [12] was due to decreased $H 19$ levels. However, here we report $>90 \%$ cell loss in shH19-LCC9 cells exposed to ICI for 3 days. This difference in results could be explained in two ways. Cook et. al., used an RNAi strategy, which as a transient methodology to knockdown ER $\alpha$ levels will be effective acutely, but after 3 days the nontransfected cells would have expanded in these cultures. We used lentiviral transduction followed by purification of the cells that were expressing the shH19 construct, using flow cytometry. Also, Cook et. al., measured cell growth using crystal violet labeling after 3 days of exposure to ICI. Crystal violet stains all live and dead cells. In our study, we used cell viability assays to measure the impact of ICI exposure to decreased H19 expression in the LCC 9 and LCC2 cells. 


\section{Cellular Physiology Cell Physiol Biochem 2018;51:1518-1532

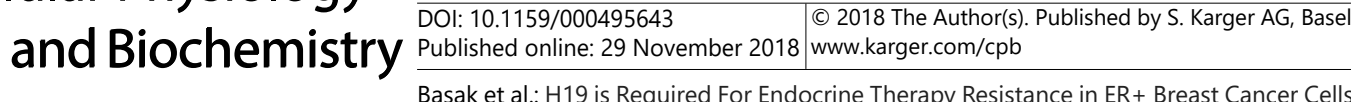

These data suggest that the ICI-resistant LCC9 cells are still reliant on ER $\alpha$-regulated cell survival signals which are presumably activated in a ligand-independent manner [9]. The ligand-independent activation of ER $\alpha$ due to somatic mutations $[29,30]$ or as yet unidentified mechanisms have made targeting ER $\alpha$ in the endocrine therapy resistant breast cancer cells very challenging. Therefore, our finding that $H 19$ regulates ER $\alpha$ gene expression in ETR cells has great clinical significance. While $H 19$ expression has been associated with tumour progression and therapy resistance in many cancers, to the best of our knowledge a role for H19 in endocrine therapy resistance has not been described before. H19 RNA consists of a micro-RNA (miR675) shown to decrease retinoblastoma gene expression [15, 31]. The exons 2-5 H19 RNA fragment has been reported to limit bioavailability of different non-coding RNAs such as Let-7A and $m i R-484[17,32]$. It is therefore inviting to hypothesize that reduced $H 19$ expression increases availability of miRs that subsequently decrease ER $\alpha$ transcript levels. A recent report suggested that $H 19$ inhibits RNA Pol II-mediated transcription by disrupting the hnRNP U-active complex [33], indicating that $H 19$ could influence the transcription of its target genes by effects on these nuclear proteins. Therefore, it is possible that $H 19$ might indirectly regulate $E R \alpha$ transcription and subsequently protein levels in the ETR cells. ICI acts as an anti-estrogen by binding to ER, resulting in an enhanced degradation via ubiquitination/proteosomal mechanism, while Tam acts as a competitive inhibitor of ER and when bound to ER causes a conformational change that inhibits the recruitment of coactivators and enhances recruitment of corepressor complexes. Here we report that in ICI and Tam-resistant breast cancer cells, decreased $H 19$ levels result in decreased ER levels, and by some as yet unknown mechanism the remaining ER proteins have become again susceptible to both ICI-induced degradation and Tam inhibitory action, leading to decreased viability of these previously therapy-resistant cells.

\section{Conclusion}

Taken together, our findings indicate that $H 19$ plays a central role in maintaining endocrine therapy resistance by modulating ER $\alpha$ expression in these cells. Moreover, decreasing $H 19$ levels using pharmacological inhibitors, that inhibit pathways regulating H19 expression in the ETR cells, helps overcome Tamoxifen and Fulvestrant-resistance.

\section{Acknowledgements}

This work was made possible through the generous funding support from the Canadian Breast Cancer Foundation (CBCF- Prairies/NWT chapter, GN\# 1452) to AR. LCM was supported by grants from the Canadian Breast Cancer Foundation (CBCF- Prairies/NWT chapter, GN\# 1213), the Canadian Cancer Society Research Institute and Canadian Institutes of Health Research. PB was funded by Manitoba Health Research Institute/ Research Manitoba Postdoctoral Fellowship. We would like to thank Monroe Chan for Flow cytometry services.

\section{Disclosure Statement}

The authors indicate no potential conflicts of interest. 


\section{Cellular Physiology Cell Physiol Biochem 2018;51:1518-1532 \begin{tabular}{l|l|l|l|} 
DOI: 10.1159/000495643 & (c) 2018 The Author(s). Published by S. Karger AG, Basel
\end{tabular} and Biochemistry Published online: 29 November 2018 www.karger.com/cpb

\section{References}

1 Ijichi N, Ikeda K, Horie-Inoue K, Inoue S: FOXP1 and estrogen signaling in breast cancer. Vitam Horm 2013;93:203-212.

-2 Ali S, Coombes RC: Estrogen receptor alpha in human breast cancer: occurrence and significance. J Mammary Gland Biol Neoplasia 2000;5:271-281.

3 Kushner PJ, Agard D, Feng WJ, Lopez G, Schiau A, Uht R, Webb P, Greene G: Oestrogen receptor function at classical and alternative response elements. Novartis Found Symp 2000;230:20-26; discussion 27-40.

$\$ 4$ Wijayaratne AL, McDonnell DP: The human estrogen receptor-alpha is a ubiquitinated protein whose stability is affected differentially by agonists, antagonists, and selective estrogen receptor modulators. J Biol Chem 2001;276:35684-35692.

5 Jordan VC: Tamoxifen: a most unlikely pioneering medicine. Nat Rev Drug Discov 2003;2:205-213.

-6 Long X, Nephew KP: Fulvestrant (ICI 182,780)-dependent interacting proteins mediate immobilization and degradation of estrogen receptor-alpha. J Biol Chem 2006;281:9607-9615.

7 Musgrove EA, Sutherland RL: Biological determinants of endocrine resistance in breast cancer. Nat Rev Cancer 2009;9:631-643.

8 Osborne CK, Schiff R: Mechanisms of endocrine resistance in breast cancer. Annu Rev Med 2011;62:233247.

-9 Brunner N, Boysen B, Jirus S, Skaar TC, Holst-Hansen C, Lippman J, Frandsen T, Spang-Thomsen M, Fuqua SA, Clarke R: MCF7/LCC9: an antiestrogen-resistant MCF-7 variant in which acquired resistance to the steroidal antiestrogen ICI 182,780 confers an early cross-resistance to the nonsteroidal antiestrogen tamoxifen. Cancer Res 1997;57:3486-3493.

10 Barone I, Brusco L, Fuqua SA: Estrogen receptor mutations and changes in downstream gene expression and signaling. Clin Cancer Res 2010;16:2702-2708.

11 Fanning SW, Mayne CG, Dharmarajan V, Carlson KE, Martin TA, Novick SJ, Toy W, Green B, Panchamukhi S, Katzenellenbogen BS, Tajkhorshid E, Griffin PR, Shen Y, Chandarlapaty S, Katzenellenbogen JA, Greene GL: Estrogen receptor alpha somatic mutations Y537S and D538G confer breast cancer endocrine resistance by stabilizing the activating function-2 binding conformation. Elife 2016;5

$\checkmark 12$ Cook KL, Clarke PA, Parmar J, Hu R, Schwartz-Roberts JL, Abu-Asab M, Warri A, Baumann WT, Clarke R: Knockdown of estrogen receptor-alpha induces autophagy and inhibits antiestrogen-mediated unfolded protein response activation, promoting ROS-induced breast cancer cell death. FASEB J 2014;28:38913905.

13 Basak P, Chatterjee S, Weger S, Bruce MC, Murphy LC, Raouf A: Estrogen regulates luminal progenitor cell differentiation through H19 gene expression. Endocr Relat Cancer 2015;22:505-517.

14 Matouk IJ, DeGroot N, Mezan S, Ayesh S, Abu-lail R, Hochberg A, Galun E: The H19 non-coding RNA is essential for human tumor growth. PloS one 2007;2:e845.

15 Berteaux N, Lottin S, Monte D, Pinte S, Quatannens B, Coll J, Hondermarck H, Curgy JJ, Dugimont T, Adriaenssens E: H19 mRNA-like noncoding RNA promotes breast cancer cell proliferation through positive control by E2F1. J Biol Chem 2005;280:29625-29636.

-16 Tsang WP, Ng EK, Ng SS, Jin H, Yu J, Sung JJ, Kwok TT: Oncofetal H19-derived miR-675 regulates tumor suppressor RB in human colorectal cancer. Carcinogenesis 2009;31:350-358.

-17 Kallen AN, Zhou XB, Xu J, Qiao C, Ma J, Yan L, Lu L, Liu C, Yi JS, Zhang H, Min W, Bennett AM, Gregory RI, Ding Y, Huang Y: The imprinted H19 lncRNA antagonizes let-7 microRNAs. Mol Cell 2013;52:101-112.

18 Ghazal S, McKinnon B, Zhou J, Mueller M, Men Y, Yang L, Mueller M, Flannery C, Huang Y, Taylor HS: H19 lncRNA alters stromal cell growth via IGF signaling in the endometrium of women with endometriosis. EMBO Mol Med 2015;7:996-1003.

19 McGuire WL: Hormone receptors: their role in predicting prognosis and response to endocrine therapy. Semin Oncol 1978;5:428-433.

20 Edwards DP: The role of coactivators and corepressors in the biology and mechanism of action of steroid hormone receptors. J Mammary Gland Biol Neoplasia 2000;5:307-324.

-21 Imren S, Fabry ME, Westerman KA, Pawliuk R, Tang P, Rosten PM, Nagel RL, Leboulch P, Eaves CJ, Humphries RK: High-level beta-globin expression and preferred intragenic integration after lentiviral transduction of human cord blood stem cells. J Clin Invest 2004;114:953-962. 


\section{Cellular Physiology Cell Physiol Biochem 2018:51:1518-1532 \begin{tabular}{ll|l} 
DOI: 10.1159/000495643 & $\begin{array}{l}\text { O } 2018 \text { The Author(s). Published by S. Karger AG, Basel } \\
\text { www.karger.com/cpb }\end{array}$
\end{tabular}

22 Chatterjee S, Basak P, Buchel E, Safneck J, Murphy LC, Mowat M, Kung SK, Eirew P, Eaves CJ, Raouf A: Breast Cancers Activate Stromal Fibroblast-Induced Suppression of Progenitors in Adjacent Normal Tissue. Stem Cell Reports 2018;10:196-211.

23 Adriaenssens E, Lottin S, Berteaux N, Hornez L, Fauquette W, Fafeur V, Peyrat JP, Le Bourhis X, Hondermarck H, Coll J, Dugimont T, Curgy JJ: Cross-talk between mesenchyme and epithelium increases H19 gene expression during scattering and morphogenesis of epithelial cells. Exp Cell Res 2002;275:215-229.

24 Vennin C, Spruyt N, Dahmani F, Julien S, Bertucci F, Finetti P, Chassat T, Bourette RP, Le Bourhis X, Adriaenssens E: H19 non coding RNA-derived miR-675 enhances tumorigenesis and metastasis of breast cancer cells by downregulating c-Cbl and Cbl-b. Oncotarget 2015;6:29209-29223.

25 Lombardo Y, Faronato M, Filipovic A, Vircillo V, Magnani L, Coombes RC: Nicastrin and Notch4 drive endocrine therapy resistance and epithelial to mesenchymal transition in MCF7 breast cancer cells. Breast Cancer Res 2014;16:R62.

-26 Simoes BM, O’Brien CS, Eyre R, Silva A, Yu L, Sarmiento-Castro A, Alferez DG, Spence K, Santiago-Gomez A, Chemi F, Acar A, Gandhi A, Howell A, Brennan K, Ryden L, Catalano S, Ando S, Gee J, Ucar A, Sims AH, Marangoni E, Farnie G, Landberg G, Howell SJ, Clarke RB: Anti-estrogen Resistance in Human Breast Tumors Is Driven by JAG1-NOTCH4-Dependent Cancer Stem Cell Activity. Cell Rep 2015;12:1968-1977.

27 Hu M, Polyak K: Microenvironmental regulation of cancer development. Curr Opin Genet Dev 2008;18:27-34.

28 Mao Y, Keller ET, Garfield DH, Shen K, Wang J: Stromal cells in tumor microenvironment and breast cancer. Cancer Metastasis Rev 2013;32:303-315.

29 Alluri PG, Speers C, Chinnaiyan AM: Estrogen receptor mutations and their role in breast cancer progression. Breast cancer research : BCR 2014;16:494.

-30 Fuqua SA, Gu G, Rechoum Y: Estrogen receptor (ER) alpha mutations in breast cancer: hidden in plain sight. Breast Cancer Res Treat 2014;144:11-19.

-31 Raveh E, Matouk IJ, Gilon M, Hochberg A: The H19 Long non-coding RNA in cancer initiation, progression and metastasis - a proposed unifying theory. Mol Cancer 2015;14:184.

-32 Zhang Q, Li X, Li X, Li X, Chen Z: LncRNA H19 promotes epithelial-mesenchymal transition (EMT) by targeting miR-484 in human lung cancer cells. J Cell Biochem 2017

-33 Wang S, Wu X, Liu Y, Yuan J, Yang F, Huang J, Meng Q, Zhou C, Liu F, Ma J, Sun S, Zheng J, Wang F: Long noncoding RNA H19 inhibits the proliferation of fetal liver cells and the Wnt signaling pathway. FEBS Lett 2016;590:559-570. 